\title{
TOPOLOGY AND MODALITY: \\ THE TOPOLOGICAL INTERPRETATION OF FIRST-ORDER MODAL LOGIC
}

\author{
STEVE AWODEY AND KOHEI KISHIDA
}

\begin{abstract}
As McKinsey and Tarski showed, the Stone representation theorem for Boolean algebras extends to algebras with operators to give topological semantics for (classical) propositional modal logic, in which the "necessity" operation is modeled by taking the interior of an arbitrary subset of a topological space. In this paper the topological interpretation is extended in a natural way to arbitrary theories of full first-order logic. The resulting system of S4 first-order modal logic is complete with respect to such topological semantics.
\end{abstract}

It has been known since the work of McKinsey and Tarski [10] that, by extending the Stone representation theorem for Boolean algebras, topological spaces provide semantics to propositional modal logic. Specifically, a necessity operator obeying the rules of the system S4 can be interpreted by the interior operation in a topological space. This result, however, is limited to propositional modal logic, and the interpretation by topological interior has never been extended to first-order modal logic. The aim of this paper is to show how such an extension can be achieved.

\section{Topological Semantics for Propositional Modal logic}

Let us review the topological semantics for propositional S4.

1.1. The System S4 of Propositional Modal Logic. Modal logic is the study of logic in which the words "necessary" and "possible" appear in statements such as

- It is necessary that the square of an integer is not negative.

- It is possible that there are more than 8 planets.

The history of modal logic is as old as that of the study of logic in general, and can be traced back to the time of Aristotle. The contemporary study of modal logic typically treats modal expressions

A grateful acknowledgment goes to the inspiring discussions with and helpful comments by Horacio Arló-Costa, Nuel Belnap, Johan van Benthem, Mark Hinchliff, Paul Hovda, Ken Manders, Eric Pacuit, Rohit Parikh, Dana Scott, and especially Guram Bezhanishvili.

Philosophy Department, Carnegie Mellon University; awodey@ $@_{\mathrm{cmu}}$. edu.

Philosophy Department, University of Pittsburgh; kok6@pitt . edu. 
as sentential operators, in the same way as $\neg$ is treated. That is, for each formula $\varphi$ of propositional logic, the following are again formulas:

$$
\begin{aligned}
& \square \varphi \quad \text { "It is necessary that } \varphi . " \\
& \diamond \varphi \quad \text { "It is possible that } \varphi . "
\end{aligned}
$$

Formulas are recursively generated from propositional letters $p, q, r, \ldots$ using the propositional operators $T, \perp, \wedge, \vee, \rightarrow, \neg$ as usual, in addition to $\square$ and $\diamond$. Hence the formulas of the language include ones such as $\square(\square p \rightarrow \diamond(\square q \wedge \neg r))$.

Among various axiom systems providing inference rules for modal operators, the system S4 of propositional modal logic consists of the rules listed below, in addition to those of classical propositional logic. Here $\varphi, \psi$ are any sentences, and $T$ is a propositional constant standing for truth (or it stands for any theorem of propositional logic if the language is not assumed to have the propositional constant). Also, define $\diamond \varphi=\neg \square \neg \varphi$.

$$
\begin{gathered}
\square \varphi \vdash \varphi \\
\square \varphi \vdash \square \square \varphi \\
\square \varphi \wedge \square \psi \vdash \square(\varphi \wedge \psi) \\
\top \vdash \square \top \\
\frac{\varphi \vdash \psi}{\square \varphi \vdash \square \psi}
\end{gathered}
$$

1.2. Topology. The S4 rules in Subsection 1.1 have been known, since McKinsey and Tarski [10], to be exactly the rules of the interior operation on topological spaces. Given a set $X$, recall that a subset $\mathcal{O}(X) \subseteq \mathcal{P}(X)$ of its powerset $\mathcal{P}(X)$ is said to be a topology on $X$ if it satisfies the following:

- $\varnothing, X \in O(X)$.

- If $U, V \in O(X)$ then $U \cap V \in O(X)$.

- If $U_{i} \in O(X)$ for all $i \in I$ then $\bigcup_{i \in I} U_{i} \in O(X)$, for any index set $I$.

Such a pair $(X, O(X))$, or often $X$ itself with $O(X)$ in mind, is called a topological space. The $U \subseteq X$ lying in $O(X)$ are called open sets of $X$, and an open set $U$ such that $a \in U$ is called a neighborhood of $a$. On the other hand, $F \subseteq X$ such that $X-F=\{x \in X \mid x \notin F\}$ is an open set is called a closed 
set. Now, given a topological space $(X, O(X))$, define an interior operation int on $\mathcal{P}(X)$ as follows: for any subset $A \subseteq X$ :

$$
\operatorname{int}(A)=\bigcup_{\substack{U \subseteq A \\ U \in O(X)}} U .
$$

Note that $\operatorname{int}(A)$ is open because the union of open sets is open. Thus $\operatorname{int}(A)$ is the largest of all open sets $U$ contained in $A$. It follows that any open set $U$ is a fixed point of int and can be written as an interior, i.e. $U=\operatorname{int}(U)$. Moreover, int obeys the following rules. For any $A, B \subseteq X$,

$$
\begin{aligned}
\operatorname{int}(A) \subseteq A \\
\operatorname{int}(A) \subseteq \operatorname{int}(\operatorname{int}(A)) \\
\operatorname{int}(A) \cap \operatorname{int}(B) \subseteq \operatorname{int}(A \cap B) \\
X \subseteq \operatorname{int}(X) \\
A \subseteq B \Longrightarrow \operatorname{int}(A) \subseteq \operatorname{int}(B)
\end{aligned}
$$

Here, if we read $A, B$ for sentences and replace $X, \cap, \subseteq$ with $\top, \wedge, \vdash$, we can see these rules are just the rules of $\mathrm{S} 4$. In a similar manner, the closure $\operatorname{cl}(A)=X-\operatorname{int}(X-A)$ of $A$, i.e. the smallest closed set containing $A$, obeys the corresponding S4 rules of $\diamond$.

1.3. Topological Semantics for Propositional S4. Let us now formally define how a language of propositional modal logic is interpreted in a topological space. Suppose we are given a language $\mathcal{L}$ of propositional modal logic and a topological space $(X, O(X))$.

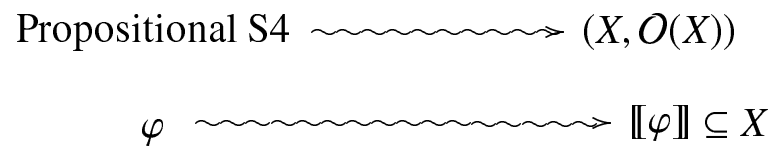

An interpretation $\llbracket \cdot]$ of $\mathcal{L}$ is a mapping from the set of sentences of $\mathcal{L}$ to $\mathcal{P}(X)$. It assigns an arbitrary subset $\llbracket p \rrbracket$ of $X$ to each atomic sentence $p$, and moreover satisfies the conditions below for connectives and operators. Here $\varphi, \psi, T$ are the same as before, while $\perp$ is either the propositional constant for falsity or any sentence whose negation is provable in propositional logic.

$$
\begin{aligned}
\llbracket \neg \varphi \rrbracket & =X-\llbracket \varphi \rrbracket, \\
\llbracket \varphi \wedge \psi \rrbracket & =\llbracket \varphi \rrbracket \cap \llbracket \psi \rrbracket, \\
\llbracket \varphi \vee \psi \rrbracket & =\llbracket \varphi \rrbracket \cup \llbracket \psi \rrbracket,
\end{aligned}
$$




$$
\begin{aligned}
\llbracket \top \rrbracket & =X, \\
\llbracket \perp \rrbracket & =\varnothing, \\
\llbracket \square \varphi \rrbracket & =\operatorname{int}(\llbracket \varphi \rrbracket) .
\end{aligned}
$$

We then write $(X, \llbracket \cdot \|) \vDash \varphi$ if the interpretation $(X, \llbracket \cdot \rrbracket)$ models a sentence $\varphi$, defined as

$$
(X, \llbracket \cdot \rrbracket) \vDash \varphi \Longleftrightarrow \llbracket \varphi \rrbracket=X .
$$

Although we can say " $\varphi$ is true in a model $(X, \| \cdot \pi)$ " to mean this relation, we should note it is not in general the case that one of $(X, \llbracket \cdot \rrbracket) \vDash \varphi$ or $(X, \llbracket \cdot \rrbracket) \vDash \neg \varphi$ must hold. Also note that $(X, \llbracket \cdot \rrbracket) \vDash \varphi$ entails $(X, \llbracket \cdot \|) \vDash \square \varphi$.

As a simple example, consider $X=[0,1]$ the closed unit interval, and $\llbracket p \rrbracket=[0,1)$ the half-open interval. Then $(X, \llbracket \cdot \rrbracket) \vDash \square(p \vee \neg p)$ because

$$
\llbracket \square(p \vee \neg p) \rrbracket=\operatorname{int}(\llbracket p \rrbracket \cup[0,1] \backslash \llbracket p \rrbracket)=\operatorname{int}([0,1])=[0,1]
$$

$\operatorname{But}(X, \llbracket \cdot \|) \not \models \square p \vee \square \neg p$, since

$$
\llbracket \square p \vee \square \neg p \rrbracket=\operatorname{int}(\llbracket p \rrbracket) \cup \operatorname{int}([0,1] \backslash \llbracket p \rrbracket)=[0,1) \neq[0,1] .
$$

With this notion of interpretation, the correspondence between the rules of Boolean operations on sets and those of the propositional connectives, and the rules of the interior operation and the S4 rules, immediately give us soundness:

Theorem 1. For any pair of sentences $\varphi, \psi$ of $\mathcal{L}$, $\varphi \vdash \psi$ is provable in $S 4 \Longrightarrow$ every topological interpretation $(X, \llbracket \cdot \rrbracket)$ has $\llbracket \varphi \rrbracket \subseteq \llbracket \psi \rrbracket$. In particular,

$$
S 4 \vdash \varphi \Longrightarrow \text { every topological interpretation }(X, \llbracket \cdot \rrbracket) \text { has }(X, \llbracket \cdot \rrbracket) \vDash \llbracket \varphi \rrbracket \text {. }
$$

The usual converse statement of completeness can be derived as a corollary of the following even stronger result.

Theorem 2 (McKinsey-Tarski, 1944). For any (consistent) theory $\mathbb{T}$ in $\mathcal{L}$ containing S4, there exist a topological space $X$ and an interpretation $\mathbb{I} \cdot \|$ such that any pair of sentences $\varphi, \psi$ of $\mathcal{L}$ satisfies 
the following:

$$
\varphi \vdash \psi \text { is provable in } \mathbb{T} \Longleftrightarrow \llbracket \varphi \rrbracket \subseteq \llbracket \psi \rrbracket \text {. }
$$

Corollary 1. For any pair $\varphi, \psi$ of sentences of $\mathcal{L}$,

$S 4$ proves $\varphi \vdash \psi \Longleftrightarrow \llbracket \varphi \rrbracket \subseteq \llbracket \psi \rrbracket$ for every topological interpretation $(X, \llbracket \cdot \rrbracket)$.

In particular,

$S 4$ proves $\vdash \varphi \Longleftrightarrow$ every topological interpretation $(X, \llbracket \cdot \rrbracket)$ has $(X, \llbracket \cdot \rrbracket) \vDash \llbracket \varphi \rrbracket$.

\section{Semantics for First-Order Logic}

The goal of this paper is to extend the topological semantics in the foregoing section to first-order modal logic. In this section we introduce some notation for the standard semantics of (non-modal) first-order logic, which will be convenient for our purposes.

2.1. Denotational Interpretation. Suppose we are given a language $\mathcal{L}$ in first-order logic. $\mathcal{L}$ has primitive relation symbols $R_{i}(i \in I)$, function symbols $f_{j}(j \in J)$, constants $c_{k}(k \in K)$. Then, as usual, a structure $M=\left\langle D, R_{i}{ }^{M}, f_{j}{ }^{M}, c_{k}{ }^{M}\right\rangle_{i \in I, j \in J, k \in K}$ for $\mathcal{L}$ consists of the following.

- A set $D$, the "domain of individuals".

- A subset $R_{i}{ }^{M} \subseteq D^{n}$ of the appropriate $n$-fold Cartesian product of the domain $D$, for each $n$-ary relation symbol $R_{i}$.

- A function $f_{j}{ }^{M}: D^{n} \rightarrow D$ for each $n$-ary function symbol $f_{j}$.

- An individual $c_{k}{ }^{M} \in D$ for each constant $c_{k}$.

Given such a structure and elements $a_{1}, \ldots, a_{n} \in D$, for any formula $\varphi\left(x_{1}, \ldots, x_{n}\right)$ with at most the displayed variables $x_{1}, \ldots, x_{n}$ free, the relation

$$
M \vDash \varphi\left[a_{1}, \ldots, a_{n}\right]
$$

of modeling a formula is recursively defined as usual.

Now we extend the "denotational" point of view to first-order languages. Whereas we gave an interpretation $\llbracket \varphi \rrbracket$ to sentences $\varphi$ in Subsection 1.3, here for first-order logic we give an interpretation also to formulas containing free variables; so we extend the notation to include interpretations

$$
\llbracket x, y \mid \varphi \rrbracket
$$


of all formulas. Here it is presupposed that no free variables appear in the formula $\varphi$ except $x, y$, but not that $x, y$ actually appear. To a sentence $\sigma$ with no free variables, we give $\llbracket \sigma \rrbracket$ as we did before. We also give interpretation $\llbracket \bar{x} \mid t \rrbracket$ to a term $t(\bar{x})$ built up from function symbols, constants and variables.

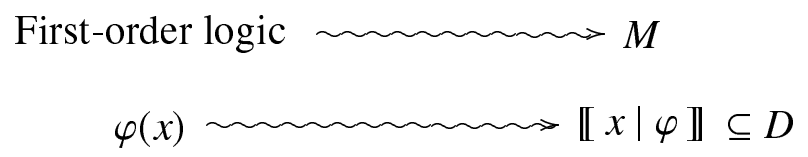

The interpretation of a formula $\varphi$ is essentially the subset of the model $M$ defined by $\varphi$ :

$$
\llbracket \bar{x} \mid \varphi \rrbracket=\left\{\bar{a} \in D^{n} \mid M \vDash \varphi[\bar{a}]\right\} \subseteq D^{n} .
$$

That is, the set of individuals satisfying $\varphi$. Then the following properties are easily derived:

$$
\begin{aligned}
\llbracket x, y \mid x=y \rrbracket & =\{(a, a) \in D \times D \mid a \in D\}, \\
\llbracket \bar{x} \mid R(\bar{x}) \rrbracket & =R^{M}, \\
\llbracket \bar{x} \mid \varphi \wedge \psi \rrbracket & =\llbracket \bar{x}|\varphi \rrbracket \cap \llbracket \bar{x}| \psi \rrbracket, \\
\llbracket \bar{x} \mid \neg \varphi \rrbracket & =D^{n}-\llbracket \bar{x} \mid \varphi \rrbracket, \\
\llbracket \bar{x} \mid \exists y \varphi \rrbracket & =\left\{\bar{a} \in D^{n}|(\bar{a}, b) \in \llbracket \bar{x}, y| \varphi \rrbracket \text { for some } b \in D\right\} .
\end{aligned}
$$

These properties could also be used as conditions to define the interpretation recursively, skipping $\vDash$ altogether. Then we would need to define $\llbracket \bar{x}, y \mid \varphi(\bar{x}) \rrbracket \subseteq D^{n+1}$ also for a formula $\varphi(\bar{x})$ which does not contain the free variable $y$, which can be done simply by

$$
\begin{aligned}
\llbracket \bar{x}, y \mid \varphi \rrbracket & =\left\{(\bar{a}, b) \in D^{n+1} \mid M \vDash \varphi[\bar{a}]\right\} \\
& =\llbracket \bar{x} \mid \varphi \rrbracket \times D .
\end{aligned}
$$

Similarly, when a term $t(\bar{x})$ has $n$ arguments, its interpretation $\llbracket \bar{x} \mid t \rrbracket$ is the function $f: D^{n} \rightarrow D$ recursively defined from $f^{M}, c^{M}$ in the expected way.

The definition of interpretation of formulas can be naturally extended to the case of $n=0$ for $D^{0}=\{*\}$, any one-element set. That is, while a subset $\llbracket \bar{x} \mid \varphi \rrbracket$ of $D^{n}$ is given for a formula $\varphi$, the interpretation of a sentence $\sigma$ is in a similar manner given as a subset $\llbracket \sigma \rrbracket$ of $D^{0}$ (a "truth value") 
as follows.

$$
\llbracket \sigma \rrbracket=\left\{* \in D^{0} \mid M \vDash \sigma\right\}= \begin{cases}1=\{*\}=D^{0} & \text { if } M \vDash \sigma, \\ 0=\varnothing \subseteq D^{0} & \text { if } M \not \models \sigma .\end{cases}
$$

Note that as in (1) we then have, for any formula $\varphi$ with at most $\bar{x}$ free,

$$
M \vDash \varphi \Longleftrightarrow \llbracket \bar{x} \mid \varphi \rrbracket=D^{n}
$$

Now, in terms of $\llbracket \cdot \rrbracket$, the usual soundness and completeness of first-order logic are expressed as follows.

Theorem 3. Given a language $\mathcal{L}$ of first-order logic, for any pair of formulas $\varphi, \psi$ of $\mathcal{L}$ with at most $\bar{x}$ free,

$$
\varphi \vdash \psi \Longleftrightarrow \text { every interpretation } M \text { has } \llbracket \bar{x}|\varphi \rrbracket \subseteq \llbracket \bar{x}| \psi \rrbracket \text {. }
$$

In particular,

$$
\vdash \varphi \Longleftrightarrow \text { every interpretation } M \text { has } M \vDash \varphi \text {. }
$$

2.2. Interpretation and Mappings. Some of the conditions which recursively define interpretation can be considered in terms of images of mappings. We sum up this fact in this subsection because it will be useful shortly. First let us introduce some notation for images. Given a mapping $f: X \rightarrow Y$ and subsets $A \subseteq X$ and $B \subseteq Y$, the direct image of $A$ and the inverse image of $B$ under $f$ shall be written respectively as follows:

$$
\begin{aligned}
f(A) & =\{f(a) \in Y \mid a \in A\}, \\
f^{-1}(B) & =\{a \in X \mid f(a) \in B\} .
\end{aligned}
$$

Next we define, for each $n$, the projection $p_{n}: D^{n+1} \rightarrow D^{n}$ to be $(\bar{a}, b) \mapsto \bar{a}$. In particular, $p_{0}: D \rightarrow D^{0}=\{*\}$ has $p_{0}(b)=*$ for all $b \in D$. Then we have

$$
\begin{aligned}
& \llbracket \bar{x} \mid \exists y \varphi \rrbracket=\left\{\bar{a} \in D^{n}|(\bar{a}, b) \in \llbracket \bar{x}, y| \varphi \rrbracket \text { for some } b \in D\right\}=p_{n}(\llbracket \bar{x}, y \mid \varphi \rrbracket), \\
& \llbracket \bar{x}, y|\psi \rrbracket=\llbracket \bar{x}| \psi \rrbracket \times D=p_{n}{ }^{-1}(\llbracket \bar{x} \mid \psi \rrbracket) .
\end{aligned}
$$



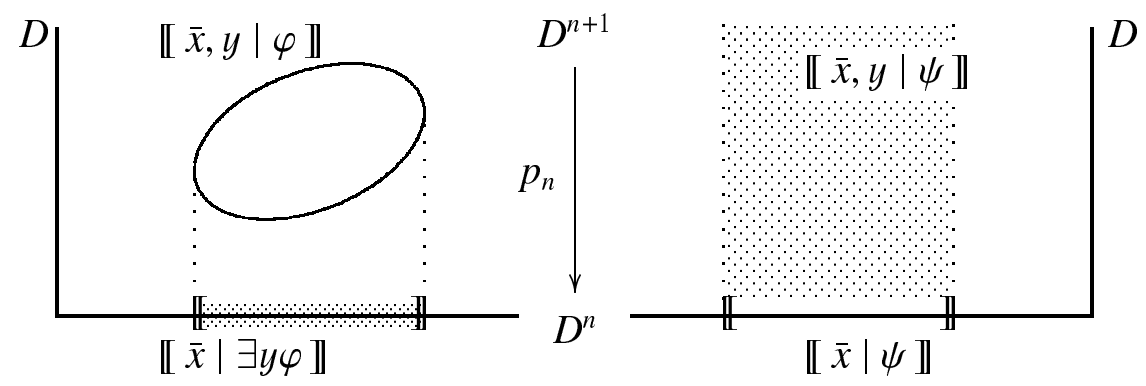

For example, $\llbracket y \mid \varphi \rrbracket$ and its image under the projection $p_{0}$, viz. $p_{0}(\llbracket y \mid \varphi \rrbracket)=\llbracket \exists y \varphi \rrbracket$, are in the relation illustrated as follows.

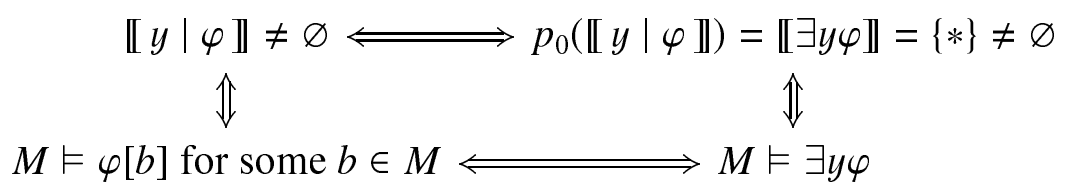

Also, because in general a mapping $f: X \rightarrow Y$ always has $f(A) \subseteq B \Longleftrightarrow A \subseteq f^{-1}(B)$, we have

$$
\llbracket \bar{x}\left|\exists y \varphi \rrbracket=p_{n}(\llbracket \bar{x}, y \mid \varphi \rrbracket) \subseteq \llbracket \bar{x}\right| \psi \rrbracket \Longleftrightarrow \llbracket \bar{x}, y\left|\varphi \rrbracket \subseteq p_{n}{ }^{-1}(\llbracket \bar{x} \mid \psi \rrbracket)=\llbracket \bar{x}, y\right| \psi \rrbracket,
$$

which corresponds to the rule $\exists y \varphi \vdash \psi \Longleftrightarrow \varphi \vdash \psi$ of first-order logic. Here the "eigenvariable" condition that $y$ does not occur freely in $\psi$ is expressed by $\llbracket \bar{x} \mid \psi \rrbracket$ making sense. ${ }^{1}$

Moreover, the substitution of terms can also be expressed by inverse images. Given a formula $\varphi(z)$ and a term $t(\bar{y})$, with the obvious notation for substitution one has:

$$
\begin{aligned}
\llbracket \bar{y} \mid \varphi(t(\bar{y})) \rrbracket & =\left\{\bar{b} \in D^{m} \mid M \vDash \varphi(t(\bar{b}))\right\} \\
& =\left\{\bar{b} \in D^{m}|\llbracket \bar{y}| t \rrbracket(\bar{b}) \in \llbracket z \mid \varphi(z) \rrbracket\right\} \\
& =\llbracket \bar{y} \mid t \rrbracket^{-1}(\llbracket z \mid \varphi(z) \rrbracket) .
\end{aligned}
$$

\section{Topological Semantics for First-Order Modal Logic}

We now extend the topological semantics reviewed in Subsection 1.3 to first-order logic. To do so, we require the notion of a sheaf over a topological space, which combines the topological semantics of propositional modal logic with the set-valued semantics of first-order logic in Section 2 , and gives a very natural semantics for first-order modal logic.

\footnotetext{
${ }^{1}$ The observation expressed here that the existential quantifier $\exists$ is left adjoint to inverse image under projection is due to Lawvere [7].
} 
3.1. Sheaves. First, recall that a map $f: X \rightarrow Y$ of topological spaces is said to be continuous if $f^{-1}(V) \subseteq X$ is open in $X$ for every open set $V \subseteq Y$. Moreover, $f$ is called a homeomorphism if $f$ has a continuous inverse map $f^{-1}: Y \rightarrow X$; and then $X$ and $Y$ are said to be homeomorphic. For a homeomorphism $f: X \rightarrow Y$, open sets $U=f^{-1}(V)$ of $X$ and $V=f(U)$ of $Y$ also correspond bijectively.

Definition 1. A sheaf over a topological space $X$ consists of a topological space $F$ and a local homeomorphism $\pi: F \rightarrow X$, meaning that every point $a$ of $F$ has some neighborhood $U \ni a$ such that $\pi(U)$ is open and the restriction $\left.\pi\right|_{U}: U \rightarrow \pi(U)$ of $\pi$ to $U$ is a homeomorphism. ${ }^{2} F$ is called the total space, and $\pi$ is called the projection from $F$ to $X$.

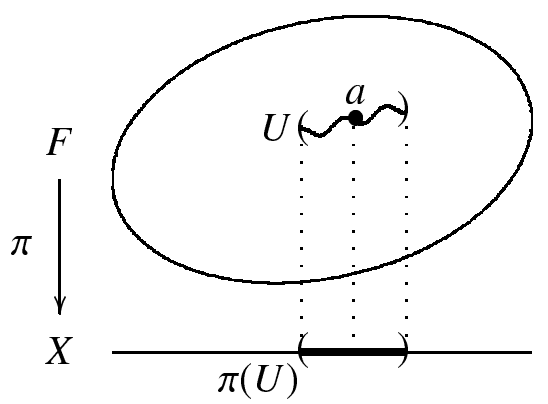

Taking a concrete example, $\mathbb{R}$ (with its usual topology) and $\pi: \mathbb{R} \rightarrow S^{1}$ such that $\pi(a)=e^{i 2 \pi a}=$ $\left(\cos 2 \pi a, \sin 2 \pi a\right.$ ) form a sheaf over the circle $S^{1}$ (with the subspace topology in $\mathbb{R}^{2}$ ). We may say that $\mathbb{R}$ draws a spiral over $S^{1}$, so that, for every $a \in \mathbb{R}$, a neighborhood $U$ small enough is homeomorphic to its image $\pi(U)$.

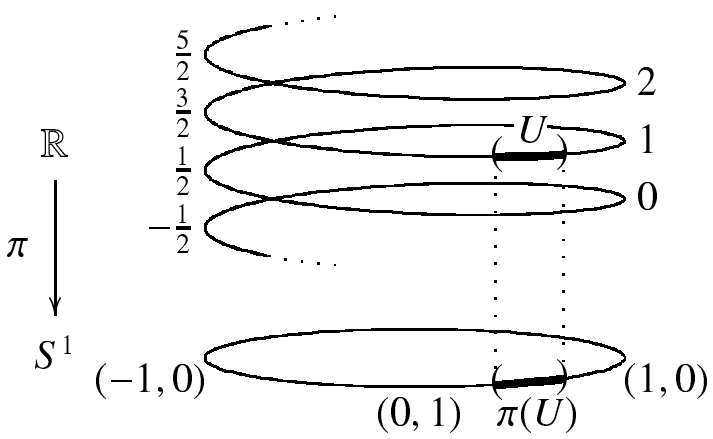

\footnotetext{
${ }^{2}$ The notion of a sheaf is sometimes defined in terms of the notion of a functor, in which case the version used here is called an étale space. The functorial notion is equivalent to the version here (in the category-theoretical sense). This paper only considers sheaves over topological spaces; but the definition using functors enables one to define sheaves more generally over various categories (see e.g. [9] for detail) and obtain more general models of modal logic.
} 
One of the properties of sheaves important for the goal of this paper is that a local homeomorphism $\pi: F \rightarrow X$ is not only continuous but is also an open map, which means that $\pi(U) \subseteq X$ is an open subset of $X$ for every open $U \subseteq F$.

It is also important that we can consider sheaves from the following viewpoint. Given a sheaf $\pi: F \rightarrow X$, take any point $p$ of $X$ and define the "stalk" $F_{p} \subseteq F$ at $p$ as follows:

$$
F_{p}=\pi^{-1}(\{p\})
$$

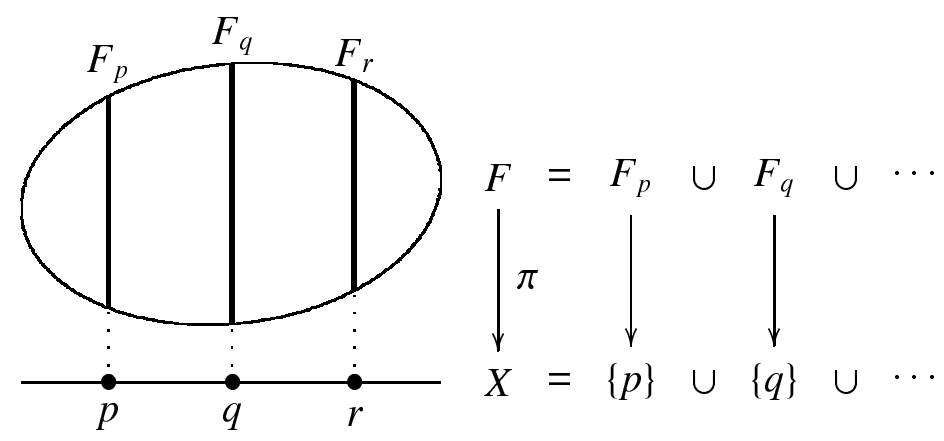

$F_{p}$ is also called the fiber of $F$ over $p$; it is shown in the figure above to be a single line over $p$. Because fibers do not intersect each other, $F$ is partitioned into fibers, so that the underlying set $|F|$ of the space $F$ can be recovered by taking the disjoint union of all fibers. That is, we can write

$$
|F|=\sum_{p \in X} F_{p},
$$

where $\sum$ indicates that the union is disjoint. By the local homeomorphism condition, each fiber $F_{p}$ forms a discrete subspace of $F$. In the context of semantics for first-order modal logic, we may think of the fibers as "possible worlds" which "change continuously" over the space $X$.

Let us mention maps of sheaves as well. A map $f$ from a sheaf $\left(F, \pi_{F}\right)$ to another $\left(G, \pi_{G}\right)$ is simply a continuous map $f: F \rightarrow G$ such that $\pi_{G} \circ f=\pi_{F}$, i.e. such that the following diagram commutes.

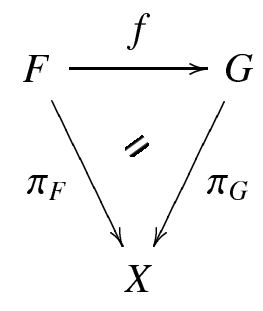


Thus $f$ respects the fibers; i.e., the underlying map $f$ can be written as a bundle of maps $f_{p}: F_{p} \rightarrow$ $G_{p}$ from fibers to fibers:

$$
f=\sum_{p \in X} f_{p}: \sum_{p \in X} F_{p} \longrightarrow \sum_{p \in X} G_{p} .
$$

It is an important fact that maps of sheaves are necessarily also local homeomorphisms, and hence are open maps.

Lastly, for a sheaf $\pi: F \rightarrow X$, the diagonal map $\Delta: F \rightarrow F \times_{X} F$ defined to be $a \mapsto(a, a)$ is a map of sheaves, and hence is an open map. ${ }^{3}$ Therefore, in particular, the image

$$
\Delta(F)=\left\{(a, a) \in F \times_{X} F \mid a \in F\right\} \subseteq F \times_{X} F
$$

of $F$ is an open subset of $F \times_{X} F$. We note that, for any topological space $F$, if $\pi: F \rightarrow X$ is an open continuous map with open diagonal map $\Delta: F \rightarrow F \times_{X} F$, then $\pi: F \rightarrow X$ is a sheaf over $X$.

3.2. Topological Semantics in Terms of Sheaves. Speaking figuratively, the extension of topological semantics using sheaves corresponds to taking the "product" of topological semantics for propositional modal logic and denotational semantics for first-order logic. The topology, or the "horizontal axis," on a space $X$ and a sheaf $F$ gives interpretation to the modal operator $\square$, and each fiber, along the "vertical axis," plays the role of a "possible world," a set providing the firstorder interpretation.

Consider a language $\mathcal{L}$ gained by adding the modal operator $\square$ to a language of first-order logic. Here, in defining formulas recursively, the usual conditions coming from first-order logic do not discriminate formulas containing modality from ones not (e.g., in the same way that $(\neg \varphi)[t / z]$, which is gained by substituting the term $t$ for the free variable $z$ in $\neg \varphi$, and $\neg(\varphi[t / z])$, by applying $\neg$ to $\varphi[t / z]$, are the same formula, we identify $(\square \varphi)[t / z]$ and $\square(\varphi[t / z])$ as the same formula $\square \varphi[t / z])$. Then, in a similar manner to Subsection 2.1, we define a structure to interpret formulas of $\mathcal{L}$ as consisting of the following. ${ }^{4}$

- A topological space $X$ and a sheaf $\pi: D \rightarrow X$ over it.

- An arbitrary subset $\llbracket R_{i} \rrbracket \subseteq D^{n}$ of the fibered product, for each $n$-ary relation symbol $R_{i}$.

- A map $\llbracket f_{j} \rrbracket: D^{n} \rightarrow D$ of sheaves, for each $n$-ary function symbol $f_{j}$.

\footnotetext{
${ }^{3} F \times_{X} F$ is the fibered product of the sheaf $F$ over the space $X$. See Appendix A for the definition of products of sheaves.

${ }^{4}$ See Appendix A for the definition of products of sheaves.
} 
- A map $\llbracket c_{k} \rrbracket: X \rightarrow D$ of sheaves from the sheaf $D^{0}=X$, for each constant $c_{k}$.

Decomposing this structure into fibers, we can see that, for each point $p \in X$, the fiber $D_{p}$ gets a standard $\mathcal{L}$-structure

$$
\left\langle D_{p}, \llbracket R_{i} \rrbracket_{p}, \llbracket f_{j} \rrbracket_{p}, \llbracket c_{k} \rrbracket_{p}\right\rangle_{i \in I, j \in J, k \in K}
$$

of first-order logic as we saw in Subsection 2.1. (Here $\llbracket R_{i} \rrbracket_{p} \subseteq D_{p}^{n_{i}}, \llbracket f_{j} \rrbracket_{p}: D_{p}^{n_{j}} \rightarrow D_{p}, \llbracket c_{k} \rrbracket_{p} \in D_{p}$.)

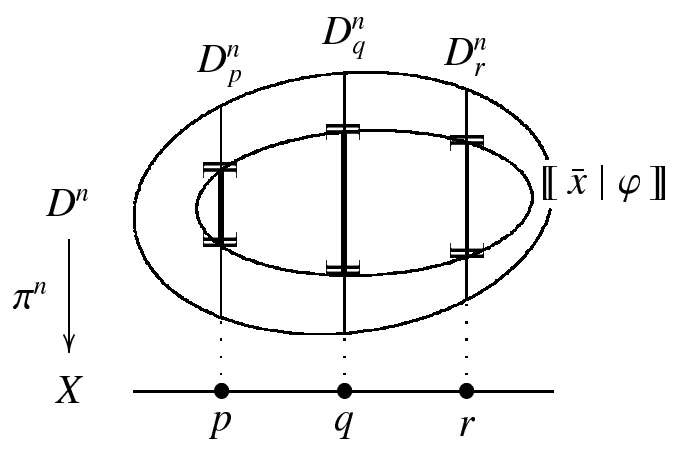

$$
\begin{array}{cccc}
\llbracket \bar{x} \mid \varphi \rrbracket & \subseteq & \left|D^{n}\right| \\
\| & & \\
\sum_{p \in X} \llbracket \bar{x} \mid \varphi \rrbracket_{p} & & \sum_{p \in X} D_{p}^{n} \\
\llbracket \bar{x} \mid \varphi \rrbracket_{p} & \subseteq & D_{p}^{n}
\end{array}
$$

So, as for the first-order part of the language, we have a fiberwise denotational interpretation $\llbracket \cdot \mathbb{1}_{p}$, each as in Subsection 2.1. We can extend the interpretation $\llbracket \cdot \rrbracket$ to all formulas $\llbracket \bar{x} \mid \varphi \rrbracket$, first fiberwise as in Subsection 2.1 to get $\llbracket \bar{x} \mid \varphi \rrbracket_{p}$, and then by "summing over $p$ ":

$$
\begin{aligned}
\llbracket \bar{x} \mid \varphi \wedge \psi \rrbracket & =\sum_{p \in X} \llbracket \bar{x} \mid \varphi \wedge \psi \mathbb{1}_{p} \\
& =\sum_{p \in X}\left(\llbracket \bar{x}\left|\varphi \rrbracket_{p} \cap \llbracket \bar{x}\right| \psi \mathbb{1}_{p}\right) \\
& =\left(\sum_{p \in X} \llbracket \bar{x} \mid \varphi \rrbracket_{p}\right) \cap\left(\sum_{p \in X} \llbracket \bar{x} \mid \psi \rrbracket_{p}\right)=\llbracket \bar{x}|\varphi \rrbracket \cap \llbracket \bar{x}| \psi \rrbracket
\end{aligned}
$$

Taking a sentence $\exists y \varphi$ for example, its interpretation is

$$
\llbracket \exists y \varphi \rrbracket=\sum_{p \in X} \llbracket \exists y \varphi \rrbracket_{p} \cong\left\{p \in X \mid \llbracket \exists y \varphi \rrbracket_{p} \neq \varnothing\right\}=\pi(\llbracket y \mid \varphi \rrbracket) \subseteq X .
$$

As can be seen in this example, the interpretation of a sentence $\sigma$ with no free variables is given as a subset of $D^{0}=X$, the "worlds" $p \in X$ at which $\sigma$ is true. 


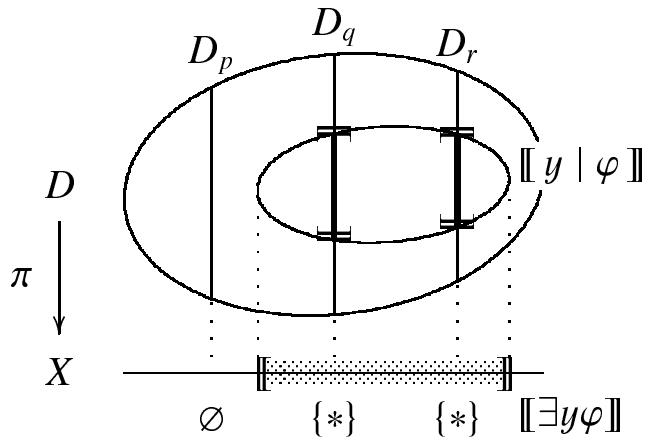

Finally, we of course use the topology of $X$ and $D$ to interpret the modal operator $\square$, i.e.,

$$
\begin{aligned}
& \llbracket \bar{x} \mid \square \varphi \rrbracket=\operatorname{int}_{D^{n}}(\llbracket \bar{x} \mid \varphi \rrbracket) \subseteq D^{n}, \\
& \llbracket \square \sigma \rrbracket=\operatorname{int}_{X}(\llbracket \sigma \rrbracket) \subseteq X .
\end{aligned}
$$

Since sentences are interpreted by subsets of $X$, we define in a similar manner to (1) and (2) of Subsections 1.3 and 2.1 as follows.

Definition 2. A formula $\varphi$ is true in an interpretation $\mathfrak{M}=(\pi: D \rightarrow X$, $\llbracket \cdot \rrbracket)$ if $\llbracket x_{1}, \ldots, x_{n} \mid \varphi \rrbracket=$ $D^{n}$; i.e.,

$$
\mathfrak{M} \vDash \varphi \Longleftrightarrow \llbracket x_{1}, \ldots, x_{n} \mid \varphi \rrbracket=D^{n}
$$

In particular, a sentence $\sigma$ is true if $\llbracket \sigma \rrbracket=X$; i.e.,

$$
\mathfrak{M} \vDash \sigma \Longleftrightarrow \llbracket \sigma \rrbracket=X
$$

Note that this specification does indeed agree with the "classical" one of McKinsey and Tarski at the level of propositional modal logic.

3.3. The System FOS4 of First-Order Modal Logic. The topological semantics given in the previous subsection is a very natural extension of the topological semantics for the system S4 of propositional modal logic to first-order logic, which can be seen from the fact that a system which is sound and complete with respect to it can be gained by simply taking the union of the axioms and rules of first-order logic and S4.

Definition 3. System FOS4 consists of the following axioms and rules.

1. All axioms and rules of (classical) first-order logic. In applying schemes, formulas containing the modal operator and ones not are not distinguished. Especially in the following 
axiom of identity, $\varphi$ may contain the modal operator.

$$
x=y \vdash \varphi(x) \rightarrow \varphi(y)
$$

2. The rules of S4 propositional modal logic. That is, for any formulas $\varphi, \psi$ and for $T$ as before,

$$
\begin{gathered}
\square \varphi \vdash \varphi \\
\square \varphi \vdash \square \square \varphi \\
\square \varphi \wedge \square \psi \vdash \square(\varphi \wedge \psi) \\
\top \vdash \square \top \\
\frac{\varphi \vdash \psi}{\square \varphi \vdash \square \psi}
\end{gathered}
$$

Listing some theorems of FOS4, not only do we have $\square \exists y \square \varphi \vdash \exists y \square \varphi$ but also the following proof is available.

$$
\frac{\frac{\square \varphi \vdash \exists y \square \varphi}{\square \square \varphi \vdash \square \exists y \square \varphi}}{\square \varphi \vdash \square \exists y \square \varphi}
$$

The last step satisfies the eigenvariable condition that $y$ does not occur freely in the right formula. In terms of the topological interpretation, this means that the image $\llbracket \bar{x} \mid \exists y \square \varphi \rrbracket$ under $p_{n}$ of an open set $\llbracket \bar{x}, y \mid \square \varphi \rrbracket$ is a fixed point of int (since $\operatorname{int}(\llbracket \bar{x} \mid \exists y \square \varphi \rrbracket)=\llbracket \bar{x}|\square \exists y \square \varphi \rrbracket=\llbracket \bar{x}| \exists y \square \varphi \rrbracket)$, i.e., it is an open set. This tells us that projections $p_{n}$ need to be open maps in order that the semantics makes FOS4 sound.

Similarly, continuity is required to model substitution so that, for any formula $\varphi(z)$ and term $t(\bar{y})$, we will have the required equality indicated by ! below:

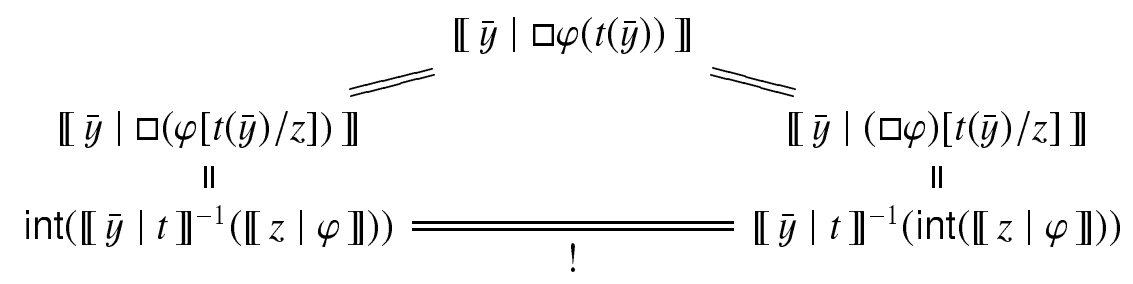


Also, by substituting $\square x=z$ for $\varphi(z)$ in the first-order axiom $x=y \vdash \varphi(x) \rightarrow \varphi(y)$ of identity, we have

$$
x=y \vdash \square x=x \rightarrow \square x=y,
$$

while $\vdash \square x=x$ is gained by the S4 rule from another axiom of identity, viz. $\vdash x=x$. Therefore $x=y \dashv \vdash x=y$ is provable. Thus the diagonal

$$
\llbracket x, y \mid x=y \rrbracket=\left\{(a, a) \in D \times_{X} D \mid a \in D\right\}=\Delta(D) \subseteq D \times_{X} D,
$$

which interprets identity, has to be open, and therefore the diagonal map $\Delta$ has to be an open map. This, together with the necessity of projections being open continuous maps, shows that the soundness of FOS4 for topological semantics actually requires the use of sheaves. Indeed, we have the following.

Theorem 4. For any formulas $\varphi$ and $\psi$,

$$
\begin{aligned}
\text { FOS4 proves } \varphi \vdash \psi \Longrightarrow & \mathbb{\llbracket} \bar{x}|\varphi \rrbracket \subseteq \llbracket \bar{x}| \psi \rrbracket \text { for every } \\
& \text { topological interpretation } \mathfrak{M}=(\pi: D \rightarrow X, \llbracket \cdot \rrbracket) .
\end{aligned}
$$

Moreover we also have completeness in the strong form of Section 1.

Theorem 5. For any (consistent) theory $\mathbb{T}$ of $\mathcal{L}$ containing FOS4, there exists a topological interpretation $\mathfrak{M}=(\pi: D \rightarrow X, \llbracket \cdot \rrbracket)$ such that, for any pair of formulas $\varphi, \psi$ of $\mathcal{L}$ with no free variables except $\bar{x}$, the following holds.

$$
\varphi \vdash \psi \text { is provable in } \mathbb{T} \Longleftrightarrow \llbracket \bar{x}|\varphi \rrbracket \subseteq \llbracket \bar{x}| \psi \rrbracket \text {. }
$$

In particular, for any sentence $\sigma$,

$$
\mathbb{T} \vdash \sigma \Longleftrightarrow \mathfrak{M} \vDash \sigma .
$$

Corollary 2. For any pair of formulas $\varphi, \psi$ of $\mathcal{L}$ with no free variables except $\bar{x}$,

$$
\llbracket \bar{x}|\varphi \rrbracket \subseteq \llbracket \bar{x}| \psi \rrbracket \text { for every topological interpretation } \mathfrak{M} \Longrightarrow \text { FOS4 proves } \varphi \vdash \psi
$$

Moreover, for any sentence $\sigma$ :

$$
\llbracket \sigma \rrbracket=X \text { for every topological interpretation } \mathfrak{M} \Longrightarrow F O S 4 \vdash \sigma \text {. }
$$

The proof of Theorem 5 is beyond the scope of this paper, but we provide a sketch as an appendix for the curious reader. 


\section{EXAMPLES OF the INTERPRETATION}

To help understand how the combination of topology and quantification works in this semantics, let us take an example of a concrete interpretation.

4.1. Necessary Properties of Individuals. Let us recall the example of a sheaf given in Subsection 3.1, i.e. the infinite helix over the circle with projection $\pi: \mathbb{R}^{+} \rightarrow S^{1}$ such that $\pi(a)=$ $(\cos 2 \pi a, \sin 2 \pi a)$, except that we now take $D=\mathbb{R}^{+}=\{a \in \mathbb{R} \mid 0<a\}$ the positive reals instead of $\mathbb{R}$. Thus we have a spiral infinitely continuing upward but with an open, downward end at 0 ; this is also a sheaf. So let $\mathfrak{M}=(\pi, \llbracket \cdot \rrbracket)$ interpret the binary relation symbol $\leqslant$ by the "no-greater-than" relation of real numbers on this sheaf, as follows:

$$
\llbracket x, y \mid x \leqslant y \rrbracket_{p}=\left\{(a, b) \in \mathbb{R}^{+2} \mid a \leqslant b \text { and } \pi(a)=\pi(b)=p\right\} .
$$

I.e., in each fiber $\mathbb{R}^{+}$, the order is just the usual one on the reals.

Then consider the truth of the following sentences under this interpretation:

$$
\begin{array}{ll}
\exists x \forall y . x \leqslant y \quad \text { "There exists } x \text { such that } x \text { is the least." } \\
\exists x \square \forall y . x \leqslant y \quad \text { "There exists } x \text { such that } x \text { is necessarily the least." }
\end{array}
$$

Now $\llbracket x \mid \forall y . x \leqslant y \rrbracket=\{a \in \mathbb{R} \mid 0<a \leqslant 1\}=(0,1]$ is the set of points of $\mathbb{R}^{+}$that are the least in their own fibers. Thus we have $\llbracket \exists x \forall y \cdot x \leqslant y \rrbracket=\pi((0,1])=S^{1}$ and (3) true in $\mathfrak{M}$. On the other hand,

$$
\llbracket x \mid \square \forall y . x \leqslant y \rrbracket=\operatorname{int}(\llbracket x \mid \forall y \cdot x \leqslant y \rrbracket)=\operatorname{int}((0,1])=\{a \in \mathbb{R} \mid 0<a<1\}=(0,1) .
$$

So $\llbracket \exists x \square \forall y . x \leqslant y \rrbracket=\pi((0,1))=S^{1}-\{(1,0)\} \neq S^{1}$, i.e., (4) is not true.

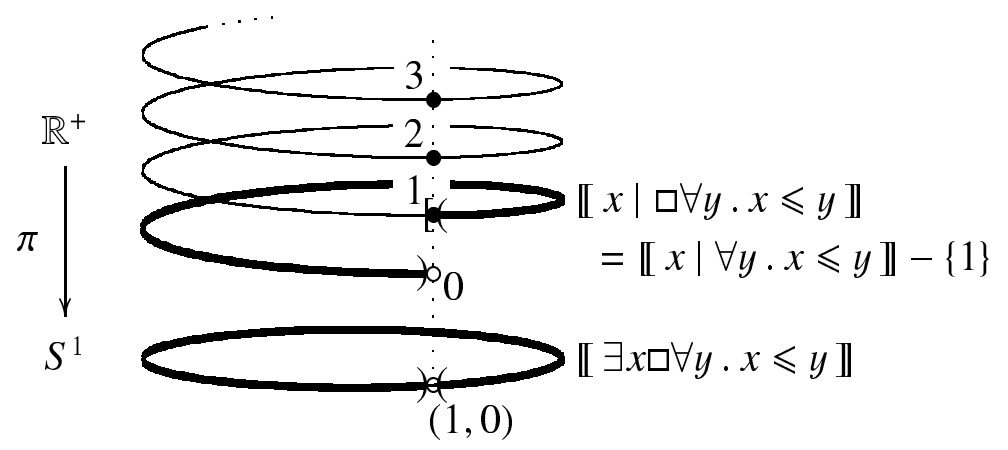

In this way, $1 \in \mathbb{R}^{+}$is "actually the least" in its fiber (or "possible world") $\mathbb{R}^{+}{ }_{(1,0)}=\{1,2,3, \ldots\}$, but not "necessarily the least." Intuitively speaking, 1 is the least in the world $\mathbb{R}_{(1,0)}^{+}$, but any 
neighborhood of this world, no matter how small a one we take, contains some world $(\{\varepsilon, 1+\varepsilon, 2+$ $\varepsilon, 3+\varepsilon, \ldots\}$ for $\varepsilon>0$ ) in which 1 is no longer the least. Note that here we used the notion " 1 in worlds near by" for explanation. Even though 1 only exists in $\mathbb{R}_{(1,0)}^{+}$, this notion still makes sense because the local homeomorphism property of the sheaf allows us to find an associated point in any other world in a sufficiently small neighborhood.

Finally, note that, because $\llbracket \exists x \forall y \cdot x \leqslant y \rrbracket=S^{1}$, we have $\llbracket \square \exists x \forall y \cdot x \leqslant y \rrbracket=\operatorname{int}(\llbracket \exists x \forall y \cdot x \leqslant y \rrbracket)=$ $\operatorname{int}\left(S^{1}\right)=S^{1}$, and so:

whence

$$
\begin{aligned}
& \mathfrak{M} \vDash \square \exists x \forall y . x \leqslant y, \\
& \mathfrak{M} \not \models \exists x \square \forall y . x \leqslant y, \\
& \mathfrak{M} \not \models \square \exists x \forall y . x \leqslant y \rightarrow \exists x \square \forall y \cdot x \leqslant y .
\end{aligned}
$$

I.e., this example provides a counter-model for a so-called "Barcan formula" of the form " $\square \exists \rightarrow$ $\exists \square ”$.

Also, note that $\llbracket x, y \mid x \leqslant y \rrbracket$ is the union of the open diagonal $\llbracket x, y \mid x=y \rrbracket$ and $\llbracket x, y \mid x<y \rrbracket$, which is open as the restriction of the open half-plain

$$
\{(a, b) \mid a, b \in \mathbb{R} \text { and } a<b\}
$$

to the fibered product $\mathbb{R}^{+2}$. Thus $\llbracket x, y \mid x \leqslant y \rrbracket$ is open, i.e., $\llbracket x, y \mid \square x \leqslant y \rrbracket=\operatorname{int}(\llbracket x, y \mid x \leqslant y \rrbracket)=$ $\llbracket x, y \mid x \leqslant y \rrbracket$. It follows that $\llbracket \exists x \forall y . \square x \leqslant y \rrbracket=\llbracket \exists x \forall y . x \leqslant y \rrbracket=S^{1}$, and so

$$
\begin{array}{ll} 
& \mathfrak{M} \vDash \exists x \forall y \square x \leqslant y, \\
& \mathfrak{M} \not \models \exists x \square \forall y . x \leqslant y, \\
\text { whence } & \mathfrak{M} \not \models \exists x \forall y \square x \leqslant y \rightarrow \exists x \square \forall y . x \leqslant y .
\end{array}
$$

I.e., $\mathfrak{M}$ is also a counter-model for the Barcan formula of the form " $\forall \square \rightarrow \square \forall$ ". (In contrast, “converse Barcan" “ $\square \forall \rightarrow \forall \square$ ” and “ $\exists \square \rightarrow \square \exists$ ” are provable in FOS4 in a similar manner to the proof in p. 14, and are valid in the topological semantics.)

4.2. Defining functions and names. In first-order logic, when a structure $\mathfrak{M}$ satisfies $\forall \bar{x} \exists ! y \varphi(\bar{x}, y)$ ("each $\bar{x}$ has a unique $y$ such that $\varphi(\bar{x}, y)$ ") a new function symbol $f_{\varphi}$ can be introduced into the language and interpreted in $\mathfrak{M}$ so that $\mathfrak{M} \vDash \forall \bar{x} \forall y\left(f_{\varphi}(\bar{x})=y \leftrightarrow \varphi(\bar{x}, y)\right)$. Does a corresponding fact hold in FOS4? 
Consider the "codiscrete" topological space consisting of two points, i.e. $X=\{p, q\}, O(X)=$ $\{X, \varnothing\}$. Moreover, consider the sheaf over $X$ consisting of two copies of $X$, i.e.,

$$
D=X \times\{0,1\}=\{(p, 0),(p, 1),(q, 0),(q, 1)\}, \quad O(D)=\{D, X \times\{0\}, X \times\{1\}, \varnothing\}
$$

with $\pi: D \rightarrow X$ defined as $(u, i) \mapsto u$. On this sheaf, let us set the interpretation of an $(n+1)$-ary relation symbol $R$ so that

$$
\llbracket \bar{x}, y\left|R(\bar{x}, y) \rrbracket_{p}=D_{p}^{n} \times\{(p, 0)\}, \quad \llbracket \bar{x}, y\right| R(\bar{x}, y) \rrbracket_{q}=D_{q}^{n} \times\{(q, 1)\}
$$

Call the model $\mathfrak{M}=\left(\pi: D \rightarrow X, R^{\mathfrak{M}}\right)$. It follows for each $u=p, q$, because $\llbracket \bar{x} \mid \exists ! y R(\bar{x}, y) \rrbracket_{u}=$ $D_{u}^{n}$, that $\mathfrak{M} \vDash \forall \bar{x} \exists ! y R(\bar{x}, y)$.

Then, however, we cannot define a function symbol $f$ satisfying $\mathfrak{M} \vDash \forall \bar{x} \forall y(f(\bar{x})=y \leftrightarrow R(\bar{x}, y))$, which entails $\mathfrak{M} \vDash \forall \bar{x} \forall y \square(f(\bar{x})=y \leftrightarrow R(\bar{x}, y))$. This is implied by the fact that $\mathfrak{M}$ does not satisfy the consequent of the theorem $\forall \bar{x} \forall y \square(f(\bar{x})=y \leftrightarrow R(\bar{x}, y)) \vdash \forall \bar{x} \exists ! y \square R(\bar{x}, y)$ of FOS4, where $\forall \bar{x} \exists ! y \square R(\bar{x}, y)$ is short for $\forall \bar{x} \exists y \forall z(y=z \leftrightarrow \square R(\bar{x}, z))$. The same thing can be expressed in terms of the interpretation as follows. The interpretation $\llbracket f \rrbracket: D^{n} \rightarrow D$ of such $f$ must satisfy

$$
\llbracket f \rrbracket(a)= \begin{cases}(p, 0) & \text { if } a \in D_{p}^{n}, \\ (q, 1) & \text { if } a \in D_{q}^{n},\end{cases}
$$

i.e., such $\llbracket f \rrbracket$ yields $\llbracket f \rrbracket^{-1}(X \times\{0\})=D_{p}^{n} \notin O\left(D^{n}\right)$ for the open subset $X \times\{0\}$ of $D$, which means $\llbracket f \rrbracket$ would not be continuous, and hence not a map of sheaves. The same thing can be said about names with $n=0$. That is, even when $\mathfrak{M} \vDash \exists ! y \varphi(y)$ holds, a name $c$ such that $\mathfrak{M} \vDash \forall y(c=y \leftrightarrow \varphi(y))$ cannot be defined in general. For example, $\mathfrak{M}$ in the previous subsection has $\exists ! x \forall y . x \leqslant y$ true, but cannot have a name for such $x$.

On the other hand, not only in this sheaf but in any interpretation $\mathfrak{M}$, a function symbol $f_{\varphi}$ can be defined so that $\mathfrak{M} \vDash \forall \bar{x} \forall y\left(f_{\varphi}(\bar{x})=y \leftrightarrow \square \varphi(\bar{x}, y)\right)$ if $\mathfrak{M} \vDash \forall \bar{x} \exists ! y \square \varphi(\bar{x}, y)$. To sum up: In FOS4, a necessary description defines a name, which then has a continuous denotation, whereas a contingent description need not have a corresponding denotation.

\section{Historical Remark}

Let us compare the topological semantics to other preceding semantics for quantified S4. To prepare ourselves for the comparison, it is very helpful to first review the relation between the following three semantics for propositional S4: 
(1) Kripke semantics in which possible worlds are preordered (i.e., connected by reflexive and transitive accessibility relation $R$ ). Propositions are subsets of the possible worlds.

(2) Topological spaces. Each point can be considered to be a possible world. Propositions are subsets of the space (as in (1)), and $\square$ is interpreted by the interior operation int.

(3) Topological Boolean (or topo-Boolean) algebras, i.e., Boolean algebras equipped with an operation int satisfying the $\mathrm{S} 4$ rules. Each point $x$ is a proposition, and the relation $x \leqslant y$ means $x$ implying $y$.

(2) and (3) are both called topological semantics. (3) is also sometimes called an algebraic semantics. (1) is (properly) subsumed by (2) by taking the Alexandroff (right) topology: let $\{y \mid x R y\}$ be a basic open set for each world $x$, and generate topology with unions of such basic open sets. (Of course, not every space is of this kind.) (2) is in turn subsumed by (3): The Boolean algebra of subsets of a space with int forms a topo-Boolean algebra, whereas McKinsey and Tarski [10] showed that any topo-Boolean algebra can occur as a subalgebra of the algebra of a space. ${ }^{5}$

Several ideas have been proposed to extend the semantics above to quantified modal logic. One is to extend (3) by completing the algebra, so that it is equipped with arbitrary meet (for $\forall$ ) and join (for $\exists$ ). This completion was shown by Rasiowa and Sikorski [11] to give a semantics with respect to which first-order $\mathrm{S} 4$ is complete.

Another idea is to extend (1) or (2) by equipping each possible world with a domain of individuals. The current notion of a Kripke sheaf derives from early work in topos theory [7, 8], and is defined to be a presheaf over a preorder $(W, R)$ (S4 Kripke frame), viz. a functor from $(W, R)$ to the category Sets of sets. That is, a Kripke sheaf $D$ over an S4 Kripke frame $(W, R)$ assigns a "domain of individuals" $D(x)$ to each world $x \in W$, and functorially provides a mapping $D_{x y}: D(x) \rightarrow D(y)$ for each $x, y \in W$ such that $x R y$; then for $a \in D(x)$ we can read $D_{x y}(a)$ to be " $a$ in the world $y$." Such a fibration of preorders can be equivalently written as follows: ${ }^{7}$ a Kripke sheaf consists of

\footnotetext{
${ }^{5}$ McKinsey and Tarski [10] showed the dual result for closure algebras.

${ }^{6}$ Such a functorial (presheaf) definition of Kripke sheaves is found e.g. in [13]. Note that $D_{x y}$ need not be an injection, whereas each $D_{x y}$ is an inclusion map in a conventioanl Kripke frame with a domain of individual.

${ }^{7}$ See Shehtman and Skvortsov [12].
} 
two S4 Kripke frames $(W, R),(D, \rho)$ and a $p$-morphism $^{8} \pi:(D, \rho) \rightarrow(W, R)$ satisfying

$$
\pi(a) R x \Longrightarrow \exists ! b[a \rho b \wedge \pi(b)=x]
$$

Then $\pi^{-1}(x) \subseteq D$ corresponds to $D(x)$. $\pi(a)$ is "the world where the individual $a$ lives," and $b$ in $(*)$ is $D_{\pi(a) x}(a)$. QS4 ${ }^{*}$ (quantified S4 with equality) is known to be complete with respect to Kripke sheaves (see e.g. [12]).

The topological semantics of this paper is the extension of (2) analogous to Kripke sheaves extending (1). In other words, the relation between (1) and (2) is preserved in the relation between Kripke sheaves and topological semantics: any Kripke sheaf $\pi:(D, \rho) \rightarrow(W, R)$ becomes a local homeomorphism by taking the Alexandroff topology both at $(W, R)$ and at $(D, \rho) .{ }^{9}$ More precisely, indeed, the category of Kripke sheaves over a preorder $P$ and monotone maps respecting fibers is exactly the topos of all sheaves over the space $P$ with the Alexandroff topology. The approach of this paper also extends the Kripke-sheaf approach by extending the interpretation to functions and names, which have been ignored in the existing semantics in terms of Kripke sheaves; ${ }^{10}$ hence the semantics is for first-order, but not just quantified, modal logic.

It is well known that local homeomorphisms over a topological space (as in (2)) are categorically equivalent to functorial sheaves over a space considered as a complete Heyting algebra (similarly to (3)), i.e. functors from the algebra to Sets satisfying certain conditions. By virtue of this fact,

\footnotetext{
${ }^{8} \mathrm{~A}$ map $\pi:(D, \rho) \rightarrow(W, R)$ of Kripke frames is called a $p$-morphism when

and

$$
a \rho b \Longrightarrow \pi(a) R \pi(b)
$$$$
\pi(a) R x \Longrightarrow \exists b[a \rho b \wedge \pi(b)=x] .
$$

are satisfied.

${ }^{9}$ The parallelism is even deeper than mentioned here. With the condition $(*)$ dropped, any $p$-morphism $\pi:(D, \rho) \rightarrow$ $(W, R)$ is called a Kripke bundle (see [12]). If semantics includes not only Kripke sheaves but also Kripke bundles, the substitution of terms is lost. In parallel to this, the substitution is lost if topological semantics includes not only sheaves (local homeomorphisms) but also bundles in general (any open continuous maps or any continuous maps).

${ }^{10}$ In the Kripke framework, Dragalin's [3] semantics dealt with functions and names, but for intuitionistic first-order logic. This logic does not require the general sheaf structure (which FOS4 or even QS4 $=$ does); instead Dragalin used Kripke frames with increasing domains (with which FOS4 and QS4 ${ }^{=}$are incomplete). In such a semantics, the identity of individuals across worlds is given, or in other words, we need not (and Dragalin did not) make explicit the fact that functions and names have to be interpreted by maps of sheaves or monotone maps. 
the semantics of this paper in terms of local homeomorphisms can also be formulated as algebraic semantics in terms of functorial sheaves, as briefly reviewed shortly in Subsection 6.

Historically, extending (3) by functorial sheaves is already suggested in [12]. ${ }^{11}$ Also, Hilken and Rydeheard [5] formulated the sheaf extension of (2), and stated its completeness as an open problem. The completeness of first-order S4 with respect to the topological semantics is first shown by the authors of this paper [1], but in the strong form of Theorem 5, i.e., the existence of a canonical model for every theory containing FOS4.

One conceptual difference between the local-homeomorphism formulation and the functorial one is that, in the former, $\square$ is interpreted by topological interior, as it was originally in McKinsey and Tarski [10]. In this sense, the local-homeomorphism semantics can be properly called the extension of McKinsey and Tarski's topological semantics. In the same way that (2) connects the three approaches (1)-(3), the topological semantics of this paper (extending (2)) subsumes Kripke sheaf semantics (the extension of (1)) on one hand, and can be seen to categorically subsume the algebraic topological semantics ([11], extending (3)) on the other hand, ${ }^{12}$ thereby giving unification to these three approaches to first-order modal logic.

\section{Background ANd Prospects}

The topological interpretation of this paper was originally formulated in terms of category and topos theory; this paper has served to reformulate it purely in terms of elementary (point-set) topology. In the original expression, we consider the geometric morphism from the topos Sets/|X| of sets indexed over a set $|X|$ to the topos $\operatorname{Sh}(X)$ of sheaves over a topological space $X$ induced by the (continuous) identity map id $:|X| \rightarrow X$. The modal operator $\square$ is interpreted by the interior operation int that the comonad $\mathrm{id}^{*} \circ \mathrm{id}_{*}$ induces on the Boolean algebra $\operatorname{Sub}_{\operatorname{Sets} /|X|}\left(\mathrm{id}^{*} F\right) \cong \mathcal{P}(F)$ of subsets of $F$. See [2] for more detail, where the equivalence between this formulation and the one in this paper is also shown.
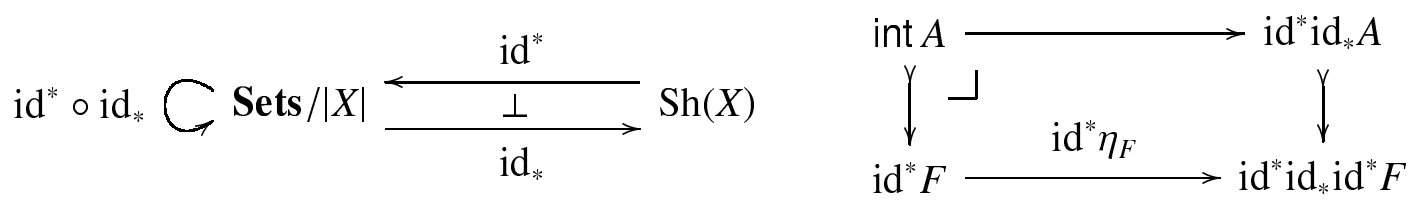

\footnotetext{
${ }^{11}$ [12], pp. 109f. There is no mention of what logic is given by the extension.

${ }^{12}$ See [4] for how such subsumption can be formulated.
} 
Although the topological formulation presented here is more elementary and perspicuous, the topos-theoretic one is more useful for generalizations. For example, we see from it that any geometric morphism of toposes (not just $\mathrm{id}^{*} \dashv \mathrm{id}_{*}$ ) induces a modality on its domain. This immediately suggests natural models for intuitionistic modal logic, typed modal logic, and higher-order modal logic.

\section{Appendix A. Products of Sheaves}

Here we review the standard definition of (fibered) products of sheaves (cf. [9]). We first need to recall some basic definitions in general topology.

Given finitely many topological spaces $X_{1}, \ldots, X_{n}$, we can introduce a topology on the cartesian product $X_{1} \times \cdots \times X_{n}$ by declaring products

$$
U_{1} \times \cdots \times U_{n} \subseteq X_{1} \times \cdots \times X_{n}
$$

of open sets $U_{1} \subseteq X_{1}, \ldots, U_{n} \subseteq X_{n}$ to be basic open sets, and thereby defining the union of any number of those basic open sets to be an open set. This topology is called the product topology.

Given a topological space $(X, O(X))$ and any subset $S \subseteq X$, we can define another topological space $(S, O(S))$, called a subspace of $(X, O(X))$ by setting:

$$
O(S)=\{U \cap S \mid U \in O(X)\}
$$

Now let us define the product of sheaves. The product of sheaves $\pi_{F}: F \rightarrow X$ and $\pi_{G}: G \rightarrow X$ is in general not the product space $F \times G$ of topological spaces $F$ and $G$; instead we take the product "over $X$," written $F \times_{X} G$. In the same way that the underlying set of a sheaf is a bundle of fibers, the underlying set of a product of sheaves is given as a bundle of products of fibers. Thus, given

$$
|F|=\sum_{p \in X} F_{p} \quad \text { and } \quad|G|=\sum_{p \in X} G_{p},
$$

we set

$$
\left|F \times_{X} G\right|=\sum_{p \in X}\left(F_{p} \times G_{p}\right)=\left\{(a, b) \in F \times G \mid \pi_{F}(a)=\pi_{G}(b)\right\} .
$$

This is called a fibered product. Since this set $\left|F \times_{X} G\right|$ is a subset of $F \times G$, we can then define the topology on $F \times{ }_{X} G$ to be the subspace topology of the product topology on $F \times G$.

The projection $\pi: F \times{ }_{X} G \rightarrow X$ (i.e., from the total space to the base space) maps $(a, b) \in F_{p} \times G_{p}$ to $p$. One can show that this projection $\pi: F \times_{X} G \rightarrow X$ is a local homeomorphism if both $\pi_{F}$ and 
$\pi_{G}$ are. We can also consider the projections $p_{F}: F \times_{X} G \rightarrow F$ and $p_{G}: F \times_{X} G \rightarrow G$ (from the product to the components), which map $(a, b) \in F_{p} \times G_{p}$ to $a \in F_{p}$ and $b \in G_{p}$ respectively. Then of course $\pi=\pi_{F} \circ p_{F}=\pi_{G} \circ p_{G}$. In sum, schematically, we have the situation:

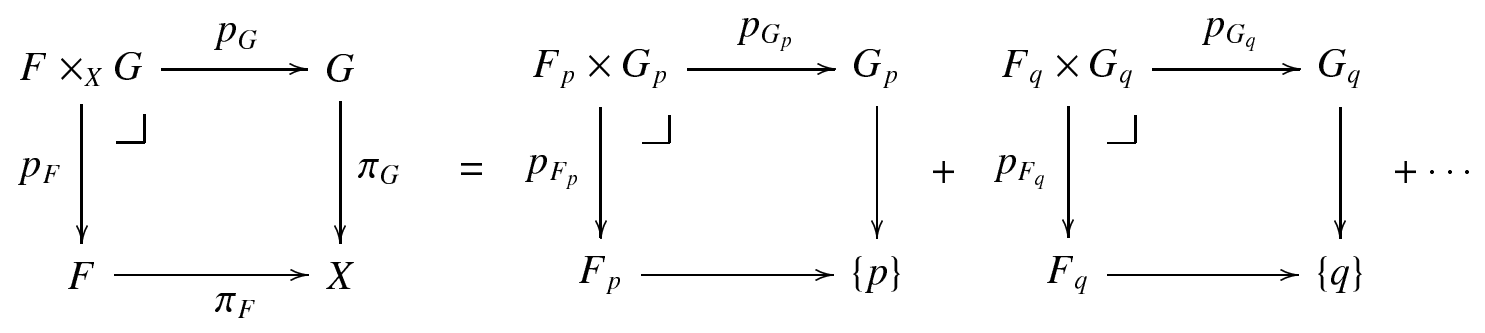

The $n$-fold product $F \times_{X} \cdots \times_{X} F$ of a sheaf $\pi: F \rightarrow X$ over $X$ is written $\pi^{n}: F^{n} \rightarrow X$. We write $F_{p}^{n}$ for the fiber $\left(F^{n}\right)_{p}=\left(F_{p}\right)^{n}$. When $n=0, F^{0}$ is $X$ itself, because the 0 -fold product of each fiber $F_{p}$ of $F$ is a singleton $F_{p}^{0}=\{*\}$ :

$$
F^{0}=\sum_{p \in X}\{*\} \cong \sum_{p \in X}\{p\}=X .
$$

Hence the projection $\pi^{0}: F^{0} \rightarrow X$ is the identity map.

\section{Appendix B. Sketch of a Completeness Proof}

Here we sketch a proof for Theorem 5, viz. the completeness of FOS4 with respect to the topological semantics. See [1] for the details.

Theorem 5. For any (consistent) theory $\mathbb{T}$ in a first-order language $\mathcal{L}$ and containing FOS4, there exists a topological interpretation $\mathfrak{M}=(\pi: D \rightarrow X, \llbracket \cdot \rrbracket)$ such that any pair of formulas $\varphi, \psi$ of $\mathcal{L}$ with no free variables except $\bar{x}$ satisfies the following:

$$
\varphi \vdash \psi \text { is provable in } \mathbb{T} \Longleftrightarrow \llbracket \bar{x}|\varphi \rrbracket \subseteq \llbracket \bar{x}| \psi \rrbracket \text {. }
$$

To sketch our proof, it is illuminating to first review a proof for the topological completeness of propositional S4, because our proof extends the essential idea of that case.

Theorem 2. For any (consistent) theory $\mathbb{T}$ in a propositional language $\mathcal{L}$ and containing $S 4$, there exists a topological interpretation $(X, \mathbb{I} \cdot \mathbb{\|})$ such that any pair of sentences $\varphi, \psi$ of $\mathcal{L}$ satisfies the following:

$$
\varphi \vdash \psi \text { is provable in } \mathbb{T} \Longleftrightarrow \llbracket \varphi \rrbracket \subseteq \llbracket \psi \rrbracket \text {. }
$$


Proof of Theorem 2 (sketch). Consider the Lindenbaum algebra $B$ of $\mathbb{T}$, which is a Boolean algebra equipped with the operation $b:[\varphi] \mapsto[\square \varphi]$. Next, take the set $\mathcal{U}$ of ultrafilters in $B$ and the Stone representation $\widehat{\cdot}: B \rightarrow \mathcal{P}(U)$. That is,

$$
\widehat{[\varphi]}=\{u \in U \mid[\varphi] \in u\}
$$

The map $\widehat{-}$ is an injective Boolean homomorphism. Next, topologize $U$ with basic open sets $\widehat{[\square \varphi}$ ] for all formulas $\varphi$. Then $\operatorname{int}(\widehat{[\varphi]})=\widehat{[\square \varphi]}$ for the interior operation int of this topology.

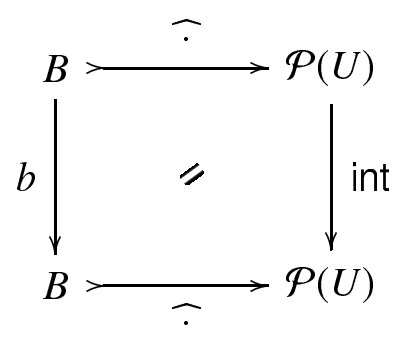

Finally, give an interpretation in $U$ to a sentence $\varphi$ by $\llbracket \varphi \rrbracket=\widehat{[\varphi]}$; this is then a topological interpretation for which we have the following:

$$
\varphi \vdash \psi \text { is provable in } \mathbb{T} \Longleftrightarrow[\varphi] \leqslant[\psi] \Longleftrightarrow \llbracket \varphi \rrbracket \subseteq \llbracket \psi \rrbracket .
$$

Note that the topology defined in the proof above coincides with the usual Stone space topology on $U$ if $\square$ is trivial, i.e. $\varphi \dashv \vdash \square$.

More importantly, we should note that each ultrafilter $u$ in $B$ can be considered a model of $\mathbb{T}$, i.e. $u \vDash \varphi$ if $\mathbb{T} \vdash \varphi$, where we write $u \vDash \varphi$ to mean $[\varphi] \in u$. In other words, the essential idea of the proof above is to take the collection of all (propositional) models of $\mathbb{T}$ and give it the topology with basic open sets defined by extensions of all $\square \varphi$.

Now, given any consistent theory $\mathbb{T}$ in a first-order modal language $\mathcal{L}$, our proof extends this key idea by first taking a sufficiently large set $\mathfrak{M}_{0}$ of first-order models of $\mathbb{T}$ in the following way. Consider the non-modal first-order language

$$
\overline{\mathcal{L}}=\mathcal{L} \cup\{\square \varphi \mid \varphi \text { is a formula of } \mathcal{L}\}
$$

given by adding to $\mathcal{L}$ an $n$-ary basic relation symbol $\square \varphi$ for each formula $\varphi$ of $\mathcal{L}$ with exactly $n$ free variables. Then Gödel's completeness theorem for first-order logic yields a class $\mathbf{M} \neq \varnothing$ of structures $M$ for $\overline{\mathcal{L}}$ such that, for any formula $\varphi$,

$$
\mathbb{T} \vdash \varphi \Longleftrightarrow M \vDash \varphi \text { for all } M \in \mathbf{M} \text {. }
$$


While $\mathbf{M}$ may be too large to be a set, the Löwenheim-Skolem theorem implies that there is a cardinal number $\lambda$ such that the set $\mathfrak{M}_{0}=\{M \in \mathbf{M} \mid\|M\| \leqslant \lambda\}$ still satisfies

$$
\mathbb{T} \vdash \varphi \Longleftrightarrow M \vDash \varphi \text { for all } M \in \mathfrak{M}_{0}
$$

This $\mathfrak{M}_{0}$, equipped with a projection $\pi: \sum_{M \in \mathfrak{M}_{0}}|M| \rightarrow \mathfrak{M}_{0}$ for $|M|$ the domains of models $M$, is our first approximation to the topological interpretation of TT required in Theorem 5.

$\mathfrak{M}_{0}$, unfortunately, cannot in general be topologized so that $\pi$ is a sheaf in the required way. To secure the necessary sheaf condition, we need to "label" $\mathfrak{M}_{0}$ so that every $a \in \sum_{M \in \mathfrak{M}_{0}}|M|$ has a name in the language. So, let us extend the language $\mathcal{L}$ to

$$
\mathcal{L}^{*}=\mathcal{L} \cup\left\{c_{i} \mid i<\lambda\right\}
$$

by adding $\lambda$-many new constant symbols. Then consider the following collection of structures for $\overline{\mathcal{L}^{*}}$ :

$$
\mathfrak{M}=\left\{M_{f} \mid M \in \mathfrak{M}_{0} \text { and } f: \lambda \rightarrow|M| \text { is a surjection }\right\},
$$

where $M_{f}$ is the expansion of $M$ to $\overline{\mathcal{L}^{*}}$ with $c_{i}^{M_{f}}=f(i)$ for all $i<\lambda$. We then have the following result for the theory $\mathbb{T}^{*}$ of $\mathfrak{M}$ : for every formula $\varphi$ of $\mathcal{L}$

$$
\mathbb{T} \vdash \varphi \Longleftrightarrow \mathbb{T}^{*} \vdash \varphi \Longleftrightarrow M_{f} \vDash \varphi \text { for each } M_{f} \in \mathfrak{M}
$$

We can then show that, if we topologize $\mathfrak{M}$ and $\sum_{M \in \mathfrak{M}}|M|$ with the extensions of $\square$ formulas as basic open sets, then $\pi: \sum_{M \in \mathfrak{M}}|M| \rightarrow \mathfrak{M}$ is a sheaf, and indeed is a topological interpretation as claimed in Theorem 5.

\section{ReFERENCES}

[1] S. Awodey and K. Kishida, “Topological Completeness for First-Order Modal Logic,” in preparation.

[2] S. Awodey and K. Kishida, "Modal Logic of Geometric Morphisms," in preparation.

[3] A. G. Dragalin, Mathematical Intuitionism: Introduction to Proof Theory, Nauka: Moscow, 1979 (in Russian); English translation by E. Mendelson, AMS, 1988.

[4] M. P. Fourman and D. S. Scott, "Sheaves and Logic," in M. P. Fourman, C. J. Mulvey, and D. S. Scott, eds., Applications of Sheaves: Proceedings of the Research Symposium on Applications of Sheaf Theory to Logic, Algebra, and Analysis, Durham, July 9-21, 1977, Springer-Verlag, 1979, pp. 302-401. 
[5] B. Hilken and D. Rydeheard, "A First Order Modal Logic and Its Sheaf Models," in M. Fairtlough, M. Mendler, and E. MogGi, eds., FLoC Satellite Workshop on Intuitionistic Modal Logics and Applications (IMLA'99), Trento, Italy, July 1999.

[6] K. Kishida, “Topological Semantics for First-Order Modal Logic,” M.Sc. Thesis, Carnegie Mellon University, 2007.

[7] F. W. Lawvere, “Adjointness in Foundations,” Dialectica 23 (1969), 281-96; reprinted in Reprints in Theory and Applications of Categories 16 (2006), 1-16.

[8] — _ "Quantifiers and Sheaves," Actes, Congrès intern. math. 1 (1970), 329-34.

[9] S. Mac Lane and I. MoerdiJK, Sheaves in Geometry and Logic: A First Introduction to Topos Theory, SpringerVerlag New York: New York, 1992.

[10] J. C. C. McKinsey and A. Tarski, “The Algebra of Topology,” Annals of Mathematics 45 (1944), $141-91$.

[11] H. Rasiowa and R. Sikorski, The Mathematics of Metamathematics, Warsaw, 1963.

[12] V. Shehtman and D. Skvortsov, "Semantics of Non-Classical First-Order Predicate Logics," in P. P. Petkov, Mathematical Logic, Plenum Press, 1990, 105-16.

[13] N.-Y. Suzuki, “Algebraic Kripke Sheaf Semantics for Non-Classical Predicate Logics,” Studia Logica 63 (1999), 387-416. 